\title{
Oscillatory Synchrony between Human Extrastriate Areas during Visual Short-Term Memory Maintenance
}

\author{
Catherine Tallon-Baudry, ${ }^{1}$ Olivier Bertrand, ${ }^{1}$ and Catherine Fischer ${ }^{2}$ \\ 1/nstitut National de la Santé et de la Recherche Médicale U280, 69003 Lyon, France, and 2Neurological Hospital, \\ 69003 Lyon, France
}

\begin{abstract}
How do we keep an object in mind? Based on evidence from animal electrophysiology and human brain-imaging techniques, it is commonly held that short-term memory relies on sustained activity in a network distributed over sensory and prefrontal cortices. How does neural firing persist in such a distributed network in the absence of visual input? Hebb's influential but so far unproved proposal, developed more than 50 years ago, is that sustained activation in short-term memory networks is maintained by reverberating activity in neuronal loops. We hypothesized that synchronized oscillatory activity, proposed to provide a dynamic link between distributed areas, could not only coordinate activity in the network but also establish reentrant loops in the system to enable both sustained firing and temporal coincidence of inputs. We show in human intracranial
\end{abstract}

Visual short-term memory is thought to rely on the coordinated activity of largely distributed networks involved in both the sensory processing and storage of visual information (GoldmanRakic, 1995; Fuster, 1997; Magnussen, 2000). Sustained neural firing was observed in the inferotemporal and prefrontal cortices of behaving monkeys during the retention of information in visual short-term memory (Fuster and Jervey, 1981; Miller et al., 1993; Wilson et al., 1993). Functional magnetic resonance imaging in humans showed that short-term memory maintenance is associated with activation of distributed regions in visual extrastriate areas and in the prefrontal cortex (for review, see Haxby et al., 2000). How is persistent firing maintained in the widely distributed short-term memory network? It has been proposed that oscillatory synchronization between spatially segregated cortical areas could provide a flexible link between the different components of a network (Singer and Gray, 1995). Such recurrent synchronous patterns are consistent with the reentrant loops postulated by Hebb (1949) to account for sustained neural activity during memory rehearsal. In Hebb's model, sustained coincident firing is necessary to enhance synaptic efficiency, a key feature enabling the transition between short- and long-term memory. Oscillatory synchrony could underlie two necessary features of Hebb's model, namely spike temporal coincidence to modify synaptic efficiency and reverberating activity to maintain a sustained firing. Although the effect of synchronous convergence of inputs on synaptic plasticity is well documented in vitro or in

\footnotetext{
Received June 15, 2001; revised July 18, 2001; accepted July 19, 2001.

We thank Jean Bullier and David Woods for their comments on an earlier version of this manuscript.

Correspondence should be addressed to Catherine Tallon-Baudry, Institut National de la Santé et de la Recherche Médicale U280, 151 cours Albert Thomas, 69003 Lyon, France. E-mail: tallon-baudry@lyon151.inserm.fr.

Copyright (C) 2001 Society for Neuroscience $0270-6474 / 01 / 210001-05 \$ 15.00 / 0$
}

recordings that limited regions of extrastriate visual areas, separated by several centimeters, become synchronized in an oscillatory mode during the rehearsal of an object in visual short-term memory. Synchrony occurs specifically in the $\beta$ range $(15-25 \mathrm{~Hz})$ and disappears in a control condition. These findings thus confirm experimentally the hypothesis of a functional role of synchronized oscillatory activity in the coordination of distributed neural activity in humans, and support Hebb's popular but unproved concept of short-term memory maintenance by reentrant activity within the activated network.

Key words: visual short-term memory; delayed-matching-tosample task; oscillations; synchrony; cell assembly; intracranial human EEG anesthetized animals (for review, see Paulsen and Sejnowski, 2000), the existence of reentrant patterns of activity during memory maintenance has not been demonstrated so far.

We intracranially recorded local neural activity in two epileptic patients who had been stereotactically implanted with multicontact depth electrodes to monitor intractable epileptic seizures; recording occurred while the patients performed a delayedmatching-to-sample task (Fig. 1). In both patients, we observed a sustained synchronized oscillatory activity in the $\beta$ range $(15-25 \mathrm{~Hz})$ during the rehearsal of the stimulus in short-term memory, between extrastriate visual areas separated by several centimeters.

\section{MATERIALS AND METHODS}

Patients. Both patients (M.B., a 48-year-old female; B.C., a 20-year-old female) suffered from pharmacologically resistant partial epilepsy and were candidates for surgery. Because the location of the epileptic focus could not be identified using noninvasive methods, they were stereotactically implanted with multicontact depth probes. Contacts were $2 \mathrm{~mm}$ long and spaced every $3.5 \mathrm{~mm}$ (center-to-center). Electrode locations were measured on x-ray images obtained in a stereotactic frame and

This article is published in The Journal of Neuroscience, Rapid Communications Section, which publishes brief, peerreviewed papers online, not in print. Rapid Communications are posted online approximately one month earlier than they would appear if printed. They are listed in the Table of Contents of the next open issue of JNeurosci. Cite this article as: JNeurosci, 2001, 21:RC177 (1-5). The publication date is the date of posting online at www.jneurosci.org.

http://www.jneurosci.org/cgi/content/full/5744 


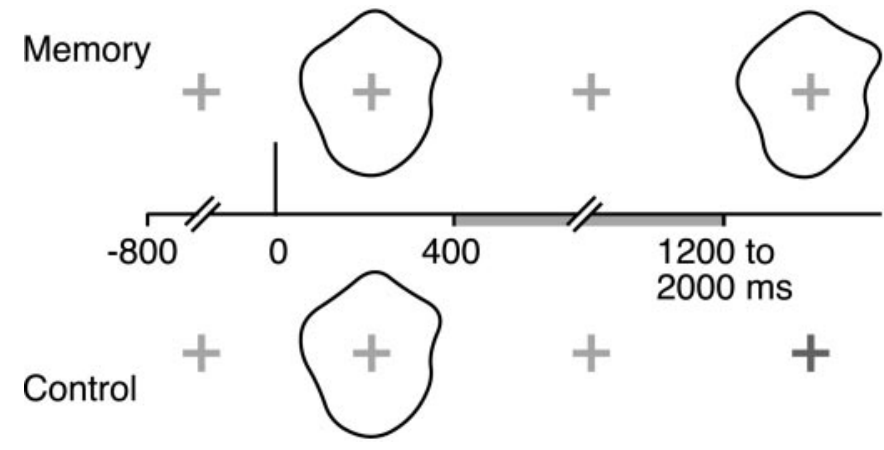

Figure 1. Block-designed paradigm. For the memory condition, subjects had to press a button when the second shape was strictly identical to the first one. Stimuli were randomly generated abstract shapes presented during $400 \mathrm{msec}$, designed to minimize verbal strategies and to foster visual imagery during the delay. For the control condition, the button press was required when the brightness of the fixation cross remained the same until the end of the delay. The difficulty of both tasks was modulated on-line to keep the subject's performance between 80 and $90 \%$ correct.

registered on structural magnetic resonance images (MRIs). The accuracy of the registration procedure was $2 \mathrm{~mm}$, estimated on another patient's MRIs obtained just after electrode explantation and in which electrode tracks were still visible. Both patients gave their informed consent to participate in the experiment. Their visual fields and acuities were normal. The signals described here were recorded away from the seizure focus (right amygdalo-hippocampus complex in patient M.B., dysplasic lesion below the right posterior calcarine sulcus in patient B.C.).

Paradigm. The experiment was divided into two conditions, a memory condition and control condition, and was run in separate blocks (Fig. 1). For the memory condition, subjects had to press a button when the second stimulus exactly matched the first one. For the control condition, they answered when the luminance of the fixation cross remained the same until the end of the trial. The intertrial interval was randomized between 2 and $3 \mathrm{sec}$. A new shape was randomly generated at each trial. For both conditions, the difficulty of the task was monitored on-line to keep the subject's performance between 80 and $90 \%$ correct. The details of stimulus construction and task difficulty monitoring have been described previously (Tallon-Baudry et al., 1998, 1999). Both patients performed the tasks as normal subjects did (Tallon-Baudry et al., 1999), at similar difficulty levels (coefficient of shape deformation between S1 and S2; M.B., 12.0; B.C., 12.9; normal subjects in the same paradigm; mean, 11.8; range, 10.5-12.6), and with comparable performances (M.B., $84 \%$ correct; B.C., $83 \%$ correct; normal subjects, $82.8 \%$ correct; range, $82.2-85.4 \%)$.

Recording and data analysis. A 64-channel continuous depth-EEG was sampled at $1000 \mathrm{~Hz}(0.1-200 \mathrm{~Hz}$ bandwidth). The electro-oculogram was monitored by surface electrodes. The video-display refresh rate was 60 $\mathrm{Hz}$ (patient M.B.) or $100 \mathrm{~Hz}$ (patient B.C.). To obtain a sufficient number of trials for statistical analysis, data from the three delay periods (until 1200,1600 , and $2000 \mathrm{msec}$ ) were pooled and analyzed up to $1200 \mathrm{msec}$ for each condition. The analysis was restricted to the electrodes located in the visual extrastriate cortex but outside of the epileptic focus (two depth probes of 10 or 15 contacts each in each patient). Because the location of the implanted electrodes depended solely on clinical requirements, the prefrontal cortex was not investigated in these patients. Raw data were visually inspected and any trial showing even small epileptic spikes was discarded, leaving 125 and 116 trials for each condition, respectively, for patient M.B. and patient B.C. There were not enough error trials to analyze them. For each single trial, bipolar derivations computed between adjacent electrode contacts were analyzed in the time-frequency domain by convolution with complex Gaussian Morlet's wavelets with a ratio $\mathrm{f} / \sigma_{\mathrm{f}}$ of 14 ; the frequency ranged from 8 to $80 \mathrm{~Hz}$ in $1 \mathrm{~Hz}$ steps (Tallon-Baudry and Bertrand, 1999). At each time $t$ and frequency $f$, the result of the convolution for trial $j$ is a complex number $A_{j}(t, f) e^{i \varphi_{j}(t, f)}$ where $A$ represents the amplitude of the signal and $\varphi$ represents its phase. Synchrony between derivations $k$ and $l$ is computed in the time-frequency domain across $n$ single trials as follows:

$$
\left\|\frac{1}{n} \sum_{j=1}^{n} e^{i\left(\varphi_{j, k}(t, f)-\varphi_{j, l}(t, f)\right)}\right\| .
$$

This synchrony factor varies between 0 (independent signals) and 1 (constant phase-lag between the two signals). It should be noted that the sign of the phase-lag measured here depends on the polarity of the signal, which in the case of bipolar derivations is arbitrary. No reliable conclusion can thus be drawn regarding the leading site. To check whether a given value of the synchrony factor could be obtained by chance, the significance of the existence of synchrony was assessed in the timefrequency domain by randomization tests (Lachaux et al., 1999) on shuffled data (5000 randomizations at each latency and frequency). This statistical method detects only episodes of synchronization that are not phase-locked to stimulus onset or offset (i.e., that appear with a jitter in latency from one trial to the next). To take into account the large number of samples tested, the significance level of 0.05 was corrected by the number of frequencies tested (72) and thus set at $p \leq 0.0006$.

\section{RESULTS}

\section{Recording sites}

The areas investigated were all located anterior to V3A (DeYoe et al., 1996; Tootell et al., 1997). In patient M.B., the most dorsal and posterior focus was in the lateral occipital sulcus and could belong to the object-responsive region located between areas V3A and MT (Tootell et al., 1996). In patient B.C., the dorsal site was located in the posterior parieto-occipital sulcus, a region found to be activated during an object memory task requiring a high degree of spatial precision (Faillenot et al., 1997). The more anterior and ventral sites were located in the fusiform gyrus (patient M.B.) and in the inferior temporal gyrus (patient B.C.). These regions have been shown to be activated during visual object perception (Gauthier et al., 1999; Ishai et al., 1999), imagery (Ishai et al., 2000), and short-term memory (Postle and D’Esposito, 1999). The Talairach coordinates (in millimeters) of the recording sites showing $\beta$ synchrony were $(+27-78+12)$ and $(+36-52-9)$ in patient M.B. and $(+18-65+18)$ and $(+61-53$ $-5)$ in patient B.C.

\section{Synchrony in the $\boldsymbol{\beta}$ range during memory retention}

Local signals were considered by computing the bipolar derivation between two adjacent electrode contacts, separated by 3.5 $\mathrm{mm}$. The existence of a significant synchrony between two bipolar derivations was computed in the time-frequency domain. In both patients, a sustained significant synchrony in the $\beta$ range (15-25 $\mathrm{Hz}$ ) could be observed during the delay period for the memory condition. This synchrony almost completely disappeared for the control condition (Figs. 2B, 3B). During the last $300 \mathrm{msec}$ of the delay (900-1200 $\mathrm{msec}$ ), the mean synchrony factor in patient M.B. at $20 \mathrm{~Hz}$ (B.C. at $16 \mathrm{~Hz}$, respectively) was 0.331 for the memory condition and 0.179 for the control condition (resp. 0.327 and 0.201 ). At the end of the delay, the synchrony factor was thus increased by $>60 \%$ in both subjects for the memory condition compared with the control and reached the significance level of 0.0006 for the memory condition only. This phenomenon was restricted to a narrow frequency range (17-24 Hz in patient M.B. and $15-17 \mathrm{~Hz}$ in patient B.C.) and could readily be observed in some single trials (Figs. 2D, 3D).

Despite the large distance between recording sites $(3.5 \mathrm{~cm}$ in patient M.B., $5.0 \mathrm{~cm}$ in patient B.C.), the mean time-lag observed in both subjects was rather short: $5.4 \mathrm{msec}$ in patient M.B. and $12.4 \mathrm{msec}$ in patient B.C. for oscillatory signals having a period of 50 and $62.5 \mathrm{msec}$, respectively. This lag was quite constant during the entire rehearsal period, with $80 \%$ of the time samples during the last $300 \mathrm{msec}$ of the delay showing lags of between -10 and 
A.

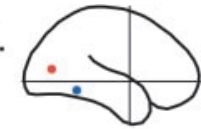

LOS

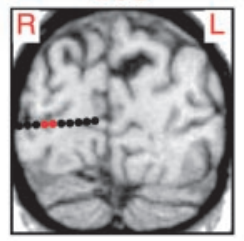

Fus

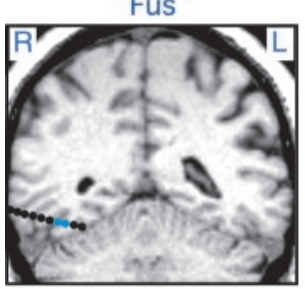

B. 80

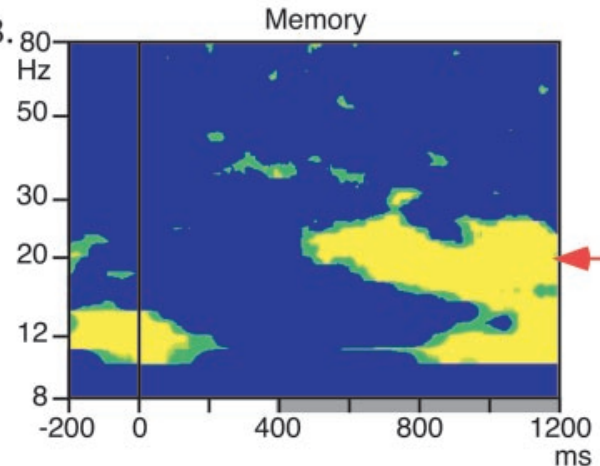

C.

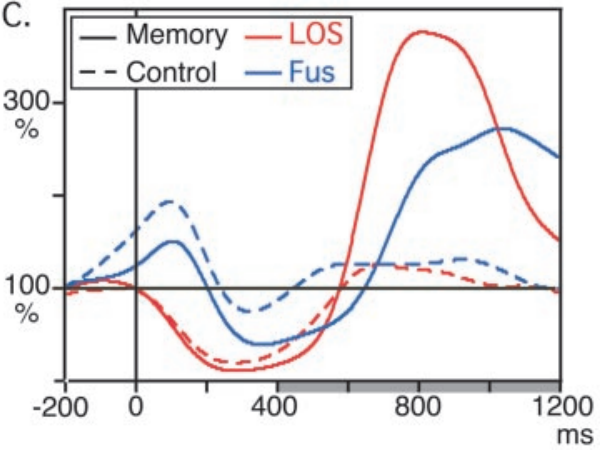

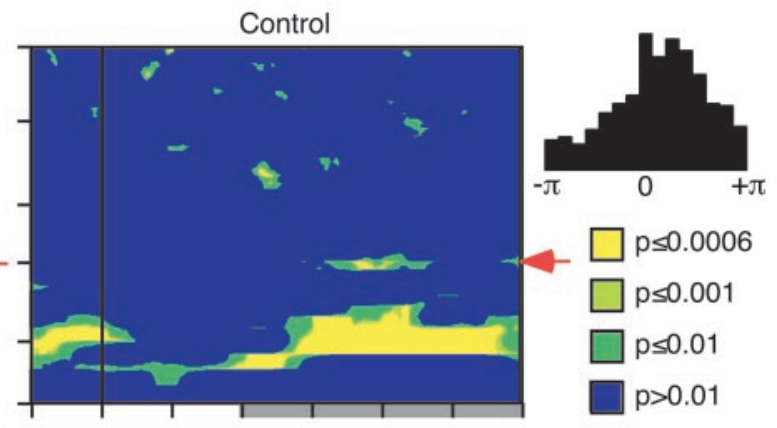
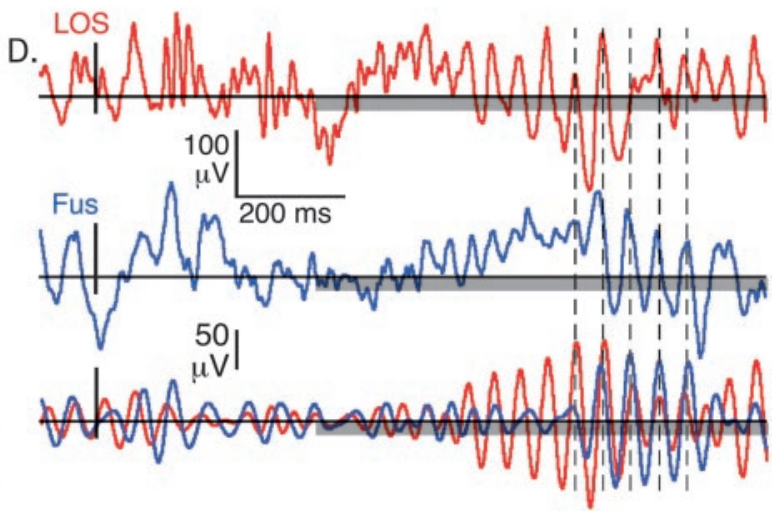

Figure 2. Patient M.B. A, Electrode location on coronal MRIs and projection on a lateral view of the brain in Talairach coordinates. $R$, Right; $L$, left; $L O S$, lateral occipital sulcus; Fus, fusiform gyrus. The bipolar derivations considered at each site are shown in red (LOS) and blue (Fus) on the MRIs. $B$, Time-frequency representations of the significance of synchrony between the two sites for the memory (left) and control (right) conditions. Time is presented on the $x$-axis; frequency is shown on the $y$-axis on a logarithmic scale. The delay period shared by all trials is indicated in gray. Synchrony around $20 \mathrm{~Hz}$ ( $\beta$ range) develops during memory rehearsal (red arrows). The distribution of the between-site phase-lag at $20 \mathrm{~Hz}$ during the last $300 \mathrm{msec}$ of the delay is presented on the rightmost panel. C. Time course of the $20 \mathrm{~Hz}$ power of local oscillations at both sites. The reference level (100\%) is taken before stimulus onset. Synchrony becomes significant at the $1 \%$ level at $468 \mathrm{msec}$ and at the $1 \%$ level at 500 msec, a latency at which the local signal power has barely begun to rise. D, Example of a single trial (top and middle rows, raw data, $0.1-200 \mathrm{~Hz}$; bottom row, superimposed $15-25 \mathrm{~Hz}$ filtered data). Synchronized $\beta$ oscillations were preceded by higher frequency oscillations in the $\gamma$ range $(30-100 \mathrm{~Hz})$ that were most prominent in the LOS.

$20 \mathrm{msec}$ at $20 \mathrm{~Hz}$ in patient M.B. and between -9 and $29 \mathrm{msec}$ at $16 \mathrm{~Hz}$ in patient B.C. (Figs. $2 B, 3 B$, rightmost panels).

An increase in between-area synchrony was not necessarily accompanied by a power increase of the local oscillations at both sites. In patient M.B., synchrony became significant for the memory condition at $p<0.0006$ at $506 \mathrm{msec}$. At this latency, the power of local oscillations at both sites was smaller for the memory condition than for the control condition (Fig. $2 C$ ). In patient B.C., the power of $\beta$ oscillations at the ventral site remained smaller for the memory condition than for the control condition during the entire delay period (Fig. $3 C$ ). The amount of between-site synchrony thus seems to be independent of the amplitude of the signal at both sites.

\section{Synchrony in other frequency bands}

Although no sustained synchrony could be observed in the $\gamma$ range during the delay, local $\gamma$ oscillations were present during stimulus presentation, although not phase-locked to stimulus onset, at frequencies that were markedly different across areas (52 $\mathrm{Hz}$ in the lateral occipital sulcus and $74 \mathrm{~Hz}$ in the fusiform gyrus in patient M.B., $60 \mathrm{~Hz}$ in the parieto-occipital sulcus and $38 \mathrm{~Hz}$ in the inferotemporal gyrus in patient B.C.). Depending on the recording site, $\beta$ oscillations either abruptly replaced $\gamma$ activity at stimulus offset or increased while $\gamma$ activity slowly decreased.

Some sustained synchrony also occurred in the $\alpha$ range (10-12 $\mathrm{Hz}$ ) in patient M.B. (Fig. 2B), but it did not vary between conditions. In the $\theta$ range $(3-6 \mathrm{~Hz})$ in patient B.C., synchrony at
$5 \mathrm{~Hz}$ became significant at the very end of the delay for the memory condition. However, $\theta$ synchrony lasted only $130 \mathrm{msec}$ for a signal at $5 \mathrm{~Hz}$ having a period of $200 \mathrm{msec}$, and thus cannot be considered as oscillatory. In addition, synchrony in the $\theta$ range does not systematically accompany $\beta$ synchrony because $\theta$ synchrony was completely absent in patient M.B. Sustained synchrony during memory rehearsal thus seems specific to the 15-25 $\mathrm{Hz}$ band, at least between the extrastriate areas investigated here.

\section{Spatial selectivity of synchrony}

The observed synchrony occurred between limited regions of cortex. It decreased when neighboring pairs of electrodes were considered that were a few millimeters away. Figure 4 shows the attenuation of the amount and significance of synchrony with lateral distance. Coupling the dorsal site with a ventral site located $14 \mathrm{~mm}$ away from the ventral site showing the maximum synchrony led to a decrease in the synchrony factor of $>50 \%$ in both patients. Moreover, $\beta$ synchrony during the delay remained significant for the neighboring derivations ( $7 \mathrm{~mm}$ away) but not further laterally in both patients. The long-distance synchrony spanning several centimeters illustrated in Figures 2 and 3 thus cannot be attributable to a common source located in between the dorsal and ventral sites and influencing both bipolar derivations by simple volume conduction effects. Instead, this synchrony occurs between small and well-delineated foci, extending over a few millimeters of cortex but separated by several centimeters. 
A.

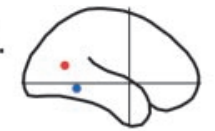

POS

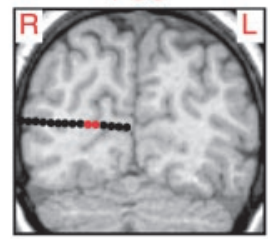

ITG

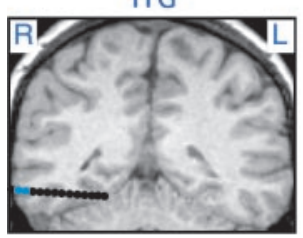

B.

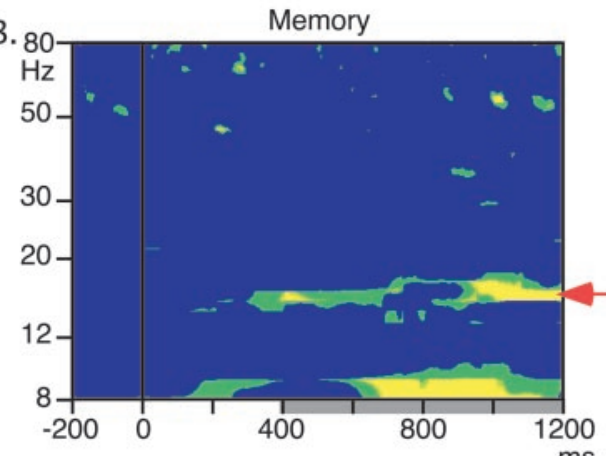

$\mathrm{ms}$

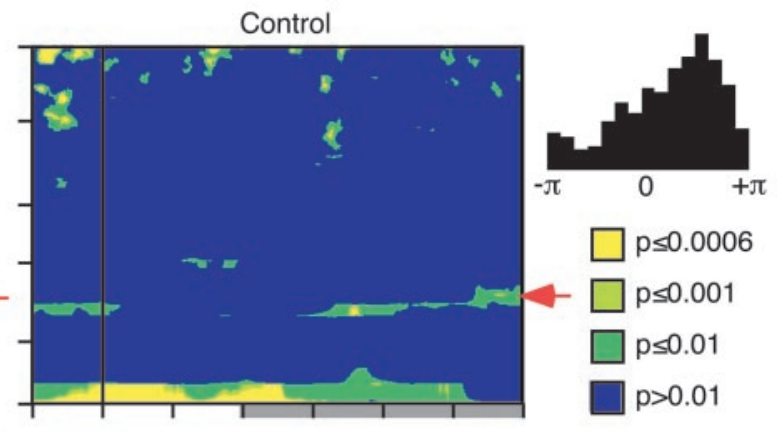

POS
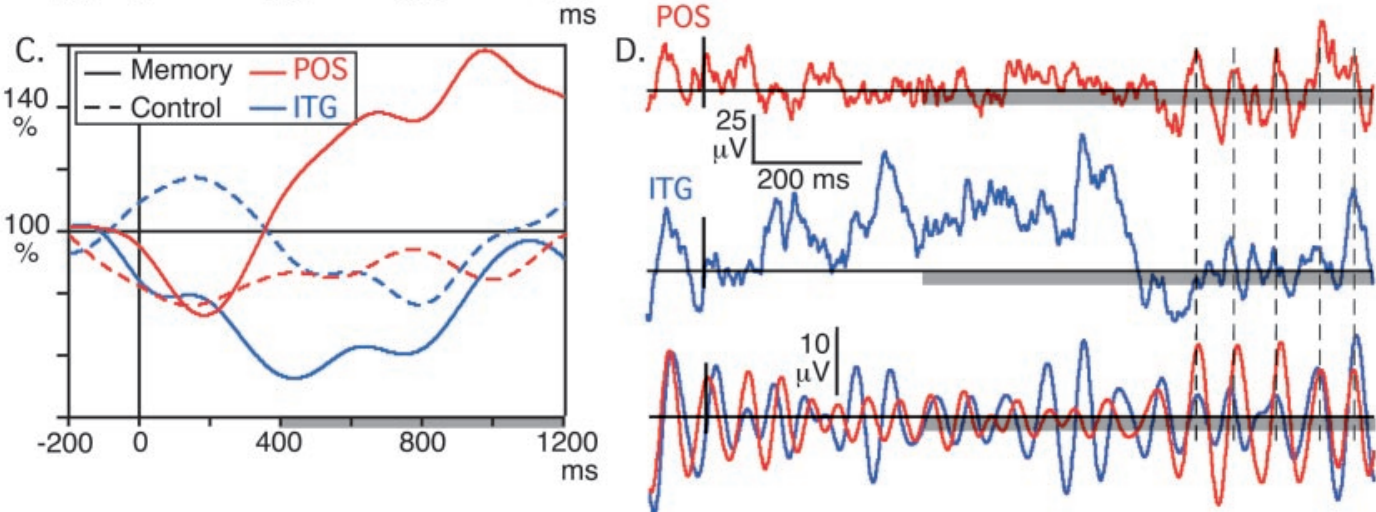

Figure 3. Patient B.C. A, Electrode location on coronal MRIs and projection on a lateral view of the brain in Talairach coordinates. $R$, Right; $L$, left; $P O S$, parieto-occipital sulcus; $I T G$, inferotemporal gyrus. The bipolar derivations considered at each site are indicated in red (POS) and blue (ITG). B, Time-frequency representations of the significance of synchrony between the two sites for the memory (left) and control (right) conditions. Synchrony develops around $16 \mathrm{~Hz}$ during the delay in the memory condition (red arrows). The distribution of the between-site phase-lag at $16 \mathrm{~Hz}$ during the last $300 \mathrm{msec}$ of the delay is shown in the rightmost panel. $C$, Time course of the $16 \mathrm{~Hz}$ power of local oscillations at both sites, for both conditions. $D$, Example of a single trial (top and middle rows, raw data, 0.1-200 Hz; bottom row, superimposed 12-20 Hz filtered data).

\section{DISCUSSION}

In both patients, synchronized oscillatory activity in the $\beta$ range between extrastriate visual areas occurred during rehearsal in memory and thus could underlie the persistent and coordinated neural activity in the short-term memory network in the absence of sensory inputs. Synchrony was present during the entire rehearsal period in patient M.B. but developed more gradually in patient B.C. Because the difficulty of both tasks was modulated on-line to keep the subject's performance between 80 and $90 \%$ correct, it seems unlikely that $\beta$ synchrony could reflect only an increased alertness level for the memory condition compared with the control condition. In addition, $\beta$ synchrony developed during the delay period only, although subjects were already highly attentive at stimulus onset. However, attentional filtering and short-term memory are strongly intermingled processes (for review, see Desimone and Duncan, 1995; Kastner and Ungerleider, 2000); for instance, to search for a face in a crowd (attentional filtering), one has to activate and maintain a description of this face (short-term memory).

Both patients were chronically treated with carbamazepine, an anticonvulsant that decreases high-frequency sustained firing through inactivation of voltage-dependent $\mathrm{Na}^{+}$channels (Loscher, 1998). The day before and on the day of the recordings, patient M.B. had to receive in addition a benzodiazepine (chlobazam) that potentiates $\mathrm{GABA}_{\mathrm{A}}$ inhibition. Both treatments may have modified the dynamics of the cellular network involved in the generation of high-frequency oscillations (Gray and McCormick, 1996; Traub et al., 1999) but are unlikely to produce focal and task-dependent increases in $\beta$ synchrony. In addition, we

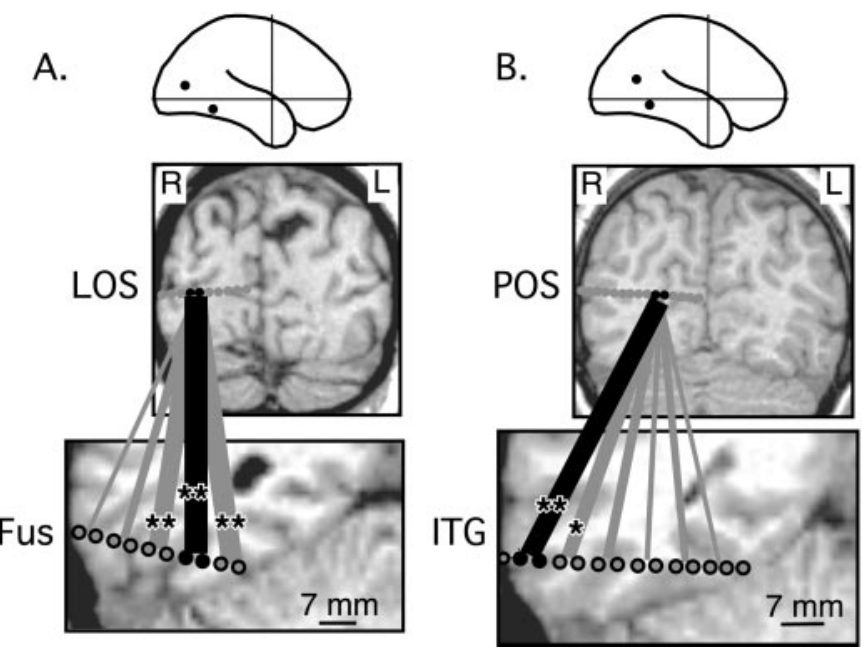

Figure 4. Spatial distribution of synchrony. The thickness of the lines connecting bipolar derivations is proportional to the amount of synchrony during the last $300 \mathrm{msec}$ of the delay in the $17-24 \mathrm{~Hz}$ band $(A$, patient M.B.) and in the $15-17 \mathrm{~Hz}$ band (B, patient B.C.). Synchrony between the pair of derivations illustrated in Figures 2 and 3 is shown in black, other pairs in gray. The significance of synchrony during the delay is indicated by asterisks $\left({ }^{*} p \leq 0.01,{ }^{* *} p \leq 0.0006\right)$. $\beta$ synchrony remains significant for the neighboring pair of electrodes but not further laterally. $R$, Right; $L$, left; LOS, lateral occipital sulcus; Fus, fusiform gyrus; POS, parietooccipital sulcus; $I T G$, inferotemporal gyrus. 
observed similar variations of $\beta$ oscillations at the scalp level (right occipital electrode) in the same paradigm in normal unmedicated subjects (Tallon-Baudry et al., 1998).

We did not find any reliable evidence for memory-related synchrony below $15 \mathrm{~Hz}$ or in the $\gamma$ range $(30-100 \mathrm{~Hz})$. Thus our results do not support directly the idea of an involvement of coupled $\theta / \gamma$ synchrony in working memory processes (Lisman and Idiart, 1995; Buzsaki, 1996; Sarnthein et al., 1998; Raghavachari et al., 2001) in the extrastriate regions explored here. Moreover, synchronization in the $\theta$ range is thought to involve the hippocampus, a structure that may not be recruited in our task, which involved only a brief retention interval, no spatial orienting, and no simultaneous memorization of several distinct items. Rather than a co-occurrence of $\gamma$ and $\theta$ oscillations, we observed a transition from local $\gamma$ oscillations to a between-area synchrony in the $\beta$ range. $\gamma$ Oscillations followed by between-site $\beta$ synchrony have also been observed in a completely different preparation (in vitro in rat hippocampal slices) after high-intensity tetanic stimulation. The tetanic stimulation induces long-lasting changes in the occurrence and synchronization of the $\beta$ oscillations, suggesting that they may be related to memory processes (Whittington et al., 1997). The local oscillations in the $\gamma$ range that we observed here could reflect the sensory processing specific to each functional area, whereas the transition to a synchronized distributed network at lower frequencies would enable the rehearsal of the global neural representation of the stimulus. If synchronous inputs in the $\beta$ range modulated the network synaptic efficiency, it may in turn promote the long-term storage of this representation.

Our results point toward a role of sustained synchronized oscillatory activity between extrastriate areas in the maintenance of an object representation in human visual short-term memory. This extends previous findings of high-frequency between-area synchrony observed in animal recordings (Bressler et al., 1993; Roelfsema et al., 1997; vonStein et al., 2000) and in human scalp EEG (Miltner et al., 1999; Rodriguez et al., 1999) concerning primarily visuomotor integration, and thus confirms experimentally the hypothesis of a role of oscillatory synchrony in the coordination of neural activity in distributed networks. Moreover, these findings may constitute the most direct evidence obtained in vivo for reverberating activity through reentrant circuits, which was postulated by Hebb (1949) a half a century ago to account for sustained activation in short-term memory networks.

\section{REFERENCES}

Bressler SL, Coppola R, Nakamura R (1993) Episodic multiregional cortical coherence at multiple frequencies during visual task performance. Nature 366:153-156.

Buzsaki G (1996) The hippocampo-neocortical dialogue. Cereb Cortex 6:81-92.

Desimone R, Duncan J (1995) Neural mechanisms of selective visual attention. Annu Rev Neurosci 18:193-222.

DeYoe EA, Carman GJ, Bandettini P, Glickman S, Wieser J, Cox R, Miller D, Neitz J (1996) Mapping striate and extrastriate visual areas in human cerebral cortex. Proc Natl Acad Sci USA 93:2382-2386.

Faillenot I, Sakata H, Costes N, Decety J, Jeannerod M (1997) Visual working memory for shape and 3D-orientation: a PET study. NeuroReport 8:859-862.

Fuster JM (1997) Network memory. Trends Neurosci 20:451-459.

Fuster JM, Jervey JP (1981) Inferotemporal neurones distinguish and retain behaviorally relevant features of visual stimuli. Science 212:952-955.

Gauthier I, Tarr MJ, Anderson AW, Skudlarski P, Gore JC (1999)
Activation of the middle fusiform "face area" increases with expertise in recognizing novel objects. Nat Neurosci 2:568-573.

Goldman-Rakic PS (1995) Cellular basis of working memory. Neuron 14:477-485.

Gray CM, McCormick DA (1996) Chattering cells: superficial pyramidal neurons contributing to the generation of synchronous oscillations in the visual cortex. Science 274:109-113.

Haxby JV, Petit L, Ungerleider LG, Courtney SM (2000) Distinguishing the functional roles of multiple regions in distributed neural systems for visual working memory. NeuroImage 11:145-156.

Hebb DO (1949) The organization of behavior. New York: Wiley

Ishai A, Ungerleider LG, Martin A, Schouten HL, Haxby JV (1999) Distributed representation of objects in the human ventral visual pathway. Proc Natl Acad Sci USA 96:9379-9384.

Ishai A, Ungerleider LG, Haxby JV (2000) Distributed neural systems for the generation of visual images. Neuron 28:979-990.

Kastner S, Ungerleider LG (2000) Mechanisms of visual attention in the human cortex. Annu Rev Neurosci 23:315-341.

Lachaux JP, Rodriguez E, Martinerie J, Varela FJ (1999) Measuring phase synchrony in brain signals. Hum Brain Mapp 8:194-208.

Lisman JE, Idiart MAP (1995) Storage of $7 \pm 2$ short-term memories in oscillatory subcycles. Science 267:1512-1515.

Loscher W (1998) New visions in the pharmacology of anticonvulsion. Eur J Pharmacol 342:1-13.

Magnussen S (2000) Low-level memory processes in vision. Trends Neurosci 23:247-251.

Miller EK, Li L, Desimone R (1993) Activity of neurons in anterior inferior temporal cortex during a short-term memory task. J Neurosci 13:1460-1478.

Miltner WHR, Braun C, Arnold M, Witte H, Taub E (1999) Coherence of $\gamma$-band EEG activity as a basis for associative learning. Nature 397:434-436.

Paulsen O, Sejnowski TJ (2000) Natural patterns of activity and longterm synaptic plasticity. Curr Opin Neurobiol 10:172-179.

Postle BR, D'Esposito M (1999) "What"-Then-"where" in visual working memory: an event-related, fMRI study. J Cogn Neurosci 11:585-597.

Raghavachari S, Kahana MJ, Rizzuto DS, Caplan JB, Kirschen MP, Bourgeois B, Madsen JR, Lisman JE (2001) Gating of human $\theta$ oscillations by a working memory task. J Neurosci 21:3175-3183.

Rodriguez E, George N, Lachaux JP, Martinerie J, Renault B, Varela FJ (1999) Perception's shadow: long-distance synchronization of human brain activity. Nature 397:430-433.

Roelfsema PR, Engel AK, Konig P, Singer W (1997) Visuomotor integration is associated with zero time-lag synchronization among cortical areas. Nature 385:157-161.

Sarnthein J, Petsche H, Rappelsberger P, Shaw GL, vonStein A (1998) Synchronization between prefrontal and posterior association cortex during human working memory. Proc Natl Acad Sci USA 95:7092-7096.

Singer W, Gray CM (1995) Visual feature integration and the temporal correlation hypothesis. Annu Rev Neurosci 18:555-586.

Tallon-Baudry C, Bertrand O (1999) Oscillatory $\gamma$ activity in humans and its role in object representation. Trends Cogn Sci 3:151-162.

Tallon-Baudry C, Bertrand O, Peronnet F, Pernier J (1998) Induced $\gamma$-band activity during the delay of a visual short-term memory task in humans. J Neurosci 18:4244-4254.

Tallon-Baudry C, Kreiter A, Bertrand O (1999) Sustained and transient oscillatory responses in the $\gamma$ and $\beta$ bands in a visual short-term memory task in humans. Vis Neurosci 16:449-459.

Tootell RBH, Dale AM, Sereno MI, Malach R (1996) New images from human visual cortex. Trends Neurosci 19:481-489.

Tootell RBH, Mendola JD, Hadjikhani NK, Ledden PJ, Liu AK, Reppas JB, Sereno MI, Dale AM (1997) Functional analysis of V3A and related areas in human visual cortex. J Neurosci 17:7060-7078.

Traub RD, Jefferys JGR, Whittington MA (1999) Fast oscillations in cortical circuits. Cambridge: MIT Press.

vonStein A, Chiang C, Konig P (2000) Top-down processing mediated by interareal synchronization. Proc Natl Acad Sci USA 97:14748-14753.

Whittington MA, Traub RD, Faulkner HJ, Stanford IM, Jefferys JGR (1997) Recurrent excitatory postsynaptic potentials induced by synchronized fast cortical oscillations. Proc Natl Acad Sci USA 94:12198-12203.

Wilson FAW, Scalaidhe SPO, Goldman-Rakic PS (1993) Dissociation of object and spatial processing domains in primate prefrontal cortex. Science 260:1955-1958. 